\title{
ATR-FTIR Spectroscopic Studies of Polymer-Based Identification Cards
}

Jasmine McGann, Georgina Sauzier, Mark J. Hackett, Simon Lewis, John McGinn, Tonya Trubshoe, Wilhelm van Bronswijk

Submitted date: 16/01/2019 - Posted date: 16/01/2019

Licence: CC BY-NC-ND 4.0

Citation information: McGann, Jasmine; Sauzier, Georgina; Hackett, Mark J.; Lewis, Simon; McGinn, John; Trubshoe, Tonya; et al. (2019): ATR-FTIR Spectroscopic Studies of Polymer-Based Identification Cards. ChemRxiv. Preprint.

Counterfeit production of polymer identity cards poses a significant economic cost to society and a threat to national security. Identifying these counterfeits is a challenge for 'frontline' personnel who lack training in specialised document examination. This study investigates the use of attenuated total reflectance Fourier Transform infrared (ATR-FTIR) spectroscopy with chemometrics as a potential approach to assessing polymer card authenticity. In situ analysis of several cards found that differentiation could be achieved based on the core polymer composition. A chemometric model was thus built for three driver's licence series produced in Western Australia and tested using a separate set of seven licences. The majority of test samples were correctly matched to the series of issue, with atypical samples recognisable based on their discriminant values. Synchrotron FTIR imaging revealed that differentiation between each series was possibly related to the adhesive used between the core layers. The approach presented in this work has the potential to be developed as a rapid screening method to identify suspect polymer cards warranting further examination.

File list (3)

IDCards_ATR_Manuscript_V1.pdf (1.08 MiB) view on ChemRxiv • download file IDCards_ATR_ESI.pdf (1.26 MiB) view on ChemRxiv • download file IDCards_ATR_GraphicalAbstract.png (540.26 KiB) view on ChemRxiv - download file 


\title{
ATR-FTIR spectroscopic studies of polymer-based identification cards
}

\author{
Jasmine McGann ${ }^{a, b \ddagger}$, Georgina Sauzier ${ }^{a, b}$, Mark J. Hackett ${ }^{a, b}$, Simon W. Lewis ${ }^{a, b *}$, John \\ McGinn ${ }^{c}$, Tonya Trubshoe ${ }^{c}$ and Wilhelm van Bronswijk ${ }^{a}$ \\ ${ }^{a}$ School of Molecular and Life Sciences, Curtin University, GPO Box U1987, Perth, Western Australia, \\ 6845, Australia. \\ ${ }^{b}$ Curtin Institute of Functional Molecules and Interfaces, Curtin University, GPO Box U1987, Perth, \\ Western Australia, 6845, Australia. \\ ${ }^{c}$ Document Examination Solutions, PO Box 7317, Karawara, Western Australia, 6152, Australia. \\ ¥ Present address: ChemCentre, PO Box 1250, Bentley, Western Australia, 6983, Australia.
}

*Author for correspondence (E-mail: S.Lewis@curtin.edu.au)

Declarations of interest: None.

\begin{abstract}
Counterfeit production of polymer identity cards poses a significant economic cost to society and a threat to national security. Identifying these counterfeits is a challenge for 'frontline' personnel who lack training in specialised document examination. This study investigates the use of attenuated total reflectance Fourier Transform infrared (ATR-FTIR) spectroscopy with chemometrics as a potential approach to assessing polymer card authenticity. In situ analysis of several cards found that differentiation could be achieved based on the core polymer composition. A chemometric model was thus built for three driver's licence series produced in Western Australia and tested using a separate set of seven licences. The majority of test samples were correctly matched to the series of issue, with atypical samples recognisable based on their discriminant values. Synchrotron FTIR imaging revealed that differentiation between each series was possibly related to the adhesive used between the core layers. The approach presented in this work has the potential to be developed as a rapid screening method to identify suspect polymer cards warranting further examination.
\end{abstract}

Keywords: Forensic science, questioned documents, infrared spectroscopy, chemometrics 


\section{Introduction}

The possession and use of polymer-based (plastic) cards has become routine in modern society. Individuals often own multiple polymer cards for various purposes including loyalty, credit or debit, insurance, health care, security access or driver's licence cards. Many of these cards can be used as a means of proving identity, gaining access to financial accounts, or obtaining high-value security documents such as passports and visas. These cards are thus a frequent target for counterfeit production, posing significant economic and security threats on an international scale $[1,2]$.

Plastic cards generally consist of a polymer core with printed information on one or both sides, covered with a laminate film. The core can be a single or multi-layer system, typically consisting of polyvinyl chloride (PVC), polyethylene terephthalate (PET), polycarbonate, or a polymer blend [3-5]. European cards frequently use polycarbonate as a core material due to its high durability, whilst PVC is thought to be the main polymer used in Australia due to its lower cost and ease of mass production [6]. The laminate can similarly be applied as a single or multi-layer system, and may consist of polyester, polycarbonate, cellulose ester, or polysulfone [7-9]. The various layers are fused through the application of heat, or using adhesives such as polyester amide, polyester urethane, or polyolefin [7]. Depending on the intended purpose of the card, additional features such as holographic images or radio frequency identification tags may also be present $[4,8]$.

Forensic examination of polymer cards largely relies on visual inspection of the printing and security features under various light sources. These examinations often make use of a stereomicroscope or specialised viewing systems, such as a video spectral comparator (VSC). Although visual examination is a widely practiced and valued method of document examination, it is heavily reliant on the training and expertise of the examiner [10]. This is challenging for 'frontline' personnel such as immigration officials or licensing agents, who do not necessarily have extensive training in questioned document examination. This may result in counterfeit cards remaining undetected at critical checkpoints. A simple, non-destructive and reliable screening tool would thus be of high value to combating this form of crime.

Vibrational spectroscopic techniques are well suited to this purpose as they provide high chemical specificity, require minimal sample preparation and are non-destructive when applied in situ. Attenuated total reflectance Fourier transform infrared (ATR-FTIR) spectroscopy is particularly appropriate for this application as it is simple, robust and readily portable. Multiple studies have demonstrated the use of ATR-FTIR spectroscopy to characterise polymer-based products such as automotive paint [11, 12], food packaging films [13-15], and textile fibres [16, 17]. However, to the best of our knowledge, the use of ATR-FTIR spectroscopy for polymer card examination has not previously been reported in the open literature.

This work used ATR-FTIR spectroscopy to identify compositional differences amongst a variety of polymer cards, and to determine whether these could act as an indicator of authenticity for driver's licence cards issued in Western Australia. The potential structural differences between cards were further investigated using synchrotron infrared microscopy. 


\section{Materials and Methods}

\subsection{Sample collection and preparation}

All polymer cards for this study were donated by individuals. Nine polymer cards issued by different organisations were used for a preliminary investigation into compositional diversity (Table 1). Subsequent investigation focussed specifically on Western Australian driver's licence cards. Three series of cards were used; those that began distribution in 2001, 2011, and 2014 (Figure S1). 15 cards were used as a calibration set for the construction of chemometric models (Table 2). An additional set of six Western Australian driver's licence cards issued from 1999-2017, as well as a New South Wales driver's licence expiring in 2017, were used as an external validation set (Table 2). Each card was thoroughly wiped with ethanol and a lint-free tissue in order to remove any contaminants prior to spectral collection.

Table 1: Polymer cards used for preliminary investigations into compositional diversity.

\begin{tabular}{ll}
\hline Card & Issuer \\
\hline ANZ Access Visa Debit & ANZ Bank, Australia \\
RAC Rewards & Royal Automotive Club, Australia \\
HBF Healthcare & HBF Health Insurance, Australia \\
Working with Children & Department of Communities, Western Australia \\
Western Australian Driver's Licence & Department of Transport, Western Australia \\
Club Sobeys & Sobeys Food Retailer, Canada \\
Saskatchewan Health Card & Government of Saskatchewan, Canada \\
Curtin Student & Curtin University, Western Australia \\
Medicare & Department of Human Services, Australia \\
\hline
\end{tabular}

Table 2: Driver's licence cards used for series investigations. $\left({ }^{*}\right)$ denotes cards used as an external validation set. $\mathrm{N} / \mathrm{A}=$ Not applicable.

\begin{tabular}{llll}
\hline Card Identifier & Series & Card Identifier & Series \\
\hline AH17 & 2014 & WVB20 & 2014 \\
JM10 & 2001 & JOM10 & 2001 \\
PC20 & 2014 & TT12 & 2001 \\
RB20 & MB14 & 2011 \\
TS12 & 2014 & AR01* & N/A (Pre-2001) \\
CM19 & 2001 & AR06* & 2001 \\
JM19 & 2011 & AR11* & 2001 \\
KVDP18 & 2011 & AR12* & 2001 \\
SL16 & 2011 & AR17* & 2011 \\
SL21 & 2001 & AR22* & 2014 \\
TL16 & 2014 & AY17* & N/A (NSW issued)
\end{tabular}

An expired 2001-series Western Australian driver's licence was used for synchrotron-source spectroscopic imaging. Microtomed cross-sections approximately $7 \mu \mathrm{m}$ thick were taken for crosssectional imaging. 


\subsection{Attenuated total reflectance infrared spectroscopy}

Spectra were obtained using a Nicolet iS50 FTIR spectrometer equipped with an iS50-ATR attachment (single-bounce diamond crystal). Measurements were taken over the $4000-400 \mathrm{~cm}^{-1}$ range in absorbance mode, with a resolution of $4 \mathrm{~cm}^{-1}$ and the co-addition of 32 scans. Data collection was performed using OMNIC software (version 9.1.24), with an air background spectrum obtained prior to each sample spectrum. As many polymer cards contain multiple layers, 'bulk' spectra were obtained from the card edge to provide representative cross-section sampling of the laminar structure (Figure S2).

\subsection{Synchrotron Fourier transform infrared imaging}

Cross-sectional mapping was completed at the infrared microspectroscopy (IRM) beamline at the Australian Synchrotron, Melbourne. The beamline consists of a Bruker Vertex 80v FTIR spectrometer and Hyperion 2000 IR microscope (Bruker Optik GmbH, Ettlingen, Germany) with a 20x objective and liquid nitrogen cooled mercury cadmium telluride (MCT) detector. High-resolution maps were collected using a germanium crystal $\left(\mathrm{n}_{\mathrm{Ge}}-4.0\right)$ ATR attachment, using a $0.5 \mathrm{~mm}$ pinhole and step size of $3 \mu \mathrm{m}$. An air background spectrum was first collected using 12 co-added scans, with sample spectra subsequently recorded with 32 co-added scans. All data collection was performed using OPUS software version 7.50 , under $19 \%$ relative humidity.

\subsection{Data analysis}

Chemometric data pre-processing and analysis was performed using the Unscrambler ${ }^{\circledR} \times 10.5$ software (Camo Software AS, Oslo, Norway). The $1880-2340 \mathrm{~cm}^{-1}$ region was omitted due to high absorbance from the diamond ATR crystal in this region. A baseline correction and range normalisation were then applied to account for spectral variations related to card thickness. Spectra were mean-centred and subjected to principal component analysis (PCA) using the non-linear iterative partial least squares algorithm. Samples were plotted using up to the first four principal components (PCs) to visualise the distribution of the samples and identify any outliers.

An LDA model was constructed for the discrimination of Western Australian driver's licences from the calibration set (12 cards), treating each series as a distinct class. This model was constructed using the linear distance measure and scores from the first four PCs (accounting for $93.0 \%$ of the variance in the dataset). The model was then used to predict spectra from the validation set (seven cards), comparing the actual and predicted classifications to evaluate the efficacy of the model.

FTIR imaging spectra were baseline corrected and analysed using Bruker OPUS v7.0 and CytoSpec 2.00.03 software (Cytospec Inc., Boston, MA, USA). Images were further processed with ImageJ 1.50i software. 


\section{Results and discussion}

\subsection{Preliminary considerations}

Spectra were initially collected from the front, back and across the edges of each card. It was decided that collection across the edge of the card was the most suitable for subsequent investigation, as it allowed a spectral cross-section of the full layer system to be obtained. However, this required the card to be manually held in contact with the ATR crystal, as no appropriate sample mount was available. Consequently, the reproducibility of the spectra must be assessed.

This was investigated by collecting spectra from a 2001 series Western Australian driver's licence card over multiple days (Figure S3). Differences in relative intensity were observed in the $3335 \mathrm{~cm}^{-1}$, $1610 \mathrm{~cm}^{-1}$ and $400 \mathrm{~cm}^{-1}$ regions, which could be attributed to differences in the contact pressure and angle of contact. The evanescent wave only penetrates $1-2 \mu \mathrm{m}$ beyond the crystal surface, making handheld contact reproducibility difficult. An additional challenge is the malleable nature of the card, allowing it to bend or slip during spectral acquisition such that parts of the laminate or other layers were no longer in contact. This may result in higher intra-group spectral variation, resulting in less distinct separation between cards using chemometric analysis.

\subsection{Population diversity}

This investigation began with the collection of spectra from a variety of polymer cards (Figure 1). Peak assignments for these spectra are presented in Table 1. All cards exhibited a shoulder at ca. $690 \mathrm{~cm}^{-1}$ corresponding to a C-Cl stretch, indicative of polyvinyl chloride (PVC). This is consistent with the proposition that PVC is the core polymer used in the manufacture of Australian identity cards. The Western Australian driver's licence, Working with Children, Medicare, Saskatchewan Health Services, Curtin Student and Club Sobeys cards also show characteristic peaks at $1450 \mathrm{~cm}^{-1}$ and $1250 \mathrm{~cm}^{-1}$. These were attributed to in-phase $\mathrm{CH}_{2}$ and $\mathrm{CH}$ bending, again associated with PVC. In the RAC, HBF Health Care and ANZ Access cards, the $\mathrm{CH}_{2}$ bending peak is shifted to ca. $1430 \mathrm{~cm}^{-1}$, denoting the presence of polyethylene terephthalate (PET). These cards also give rise to a C-C-O stretching band at $1240 \mathrm{~cm}^{-1}$ associated with PET, and a grouping of peaks in the $1100-960 \mathrm{~cm}^{-1}$ region associated with long-chain stretches in both PVC and PET. Based on this visual inspection, two groupings are evident based on the use of different polymers for their core. The RAC, HBF and ANZ cards appeared to consist of a PVC and PET blend, while the remaining cards were thought to be solely PVC-based.

Table 1: ATR-FTIR peak assignments for nine different polymer cards [18-20].

\begin{tabular}{lll}
\hline Wavenumber $\left(\mathrm{cm}^{-1}\right)$ & Vibration & Polymer \\
\hline $2930-2920 \mathrm{~cm}^{-1}$ & Symmetric C-H stretch & PVC and PET \\
$2900-2850 \mathrm{~cm}^{-1}$ & Asymmetric C-H stretch & PVC and PET \\
1450 & In-phase $\mathrm{CH}_{2}$ bend & PVC \\
1430 & $\mathrm{CH}_{2}$ bend & PET \\
1250 & In-phase CH bend & PVC \\
1240 & $\mathrm{C}-\mathrm{C}-\mathrm{O}$ & PET \\
$1100-960$ & Chain stretch & PVC and PET \\
690 & $\mathrm{C}-\mathrm{Cl}$ & PVC \\
\hline
\end{tabular}



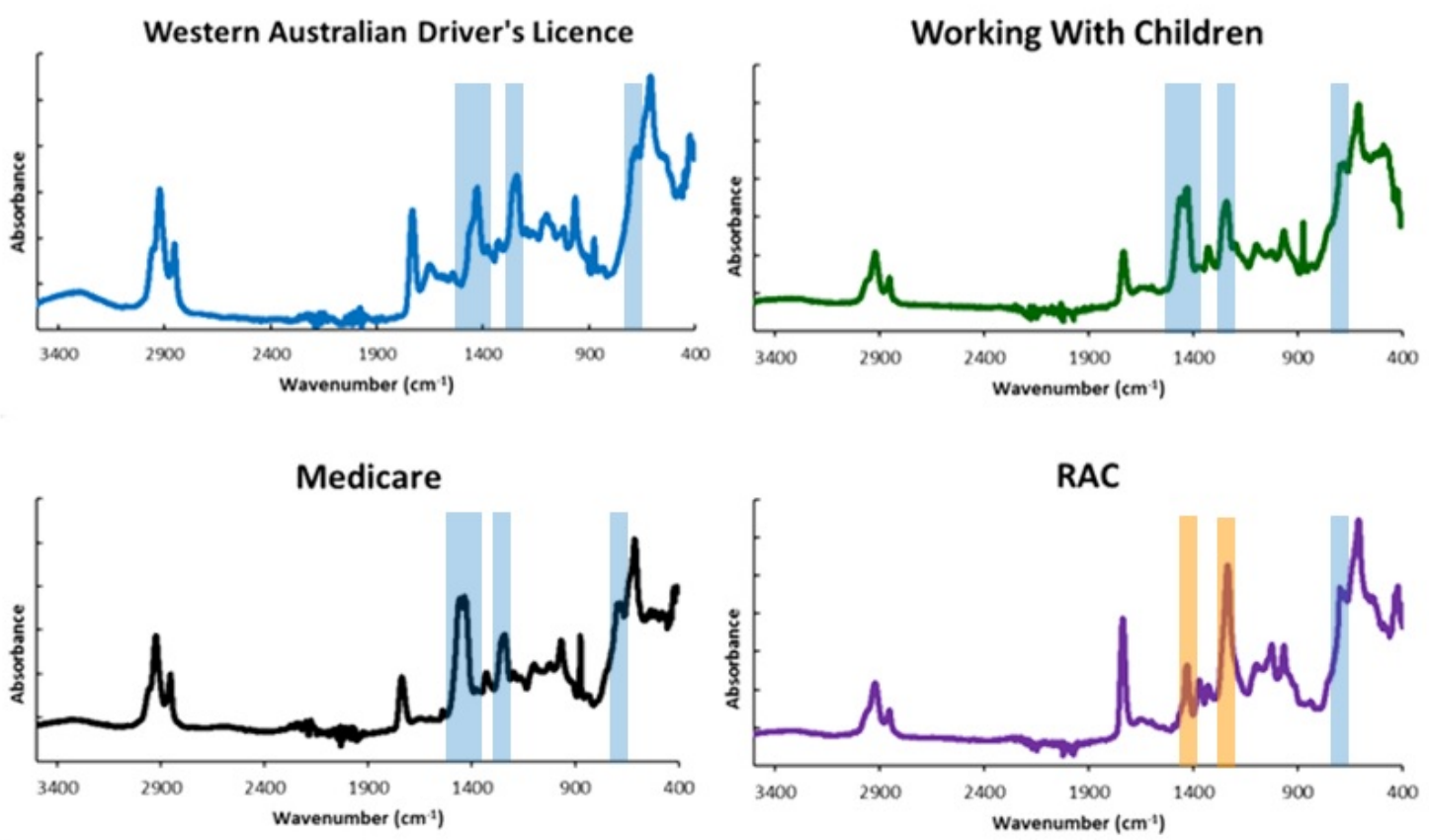

HBF Health Care
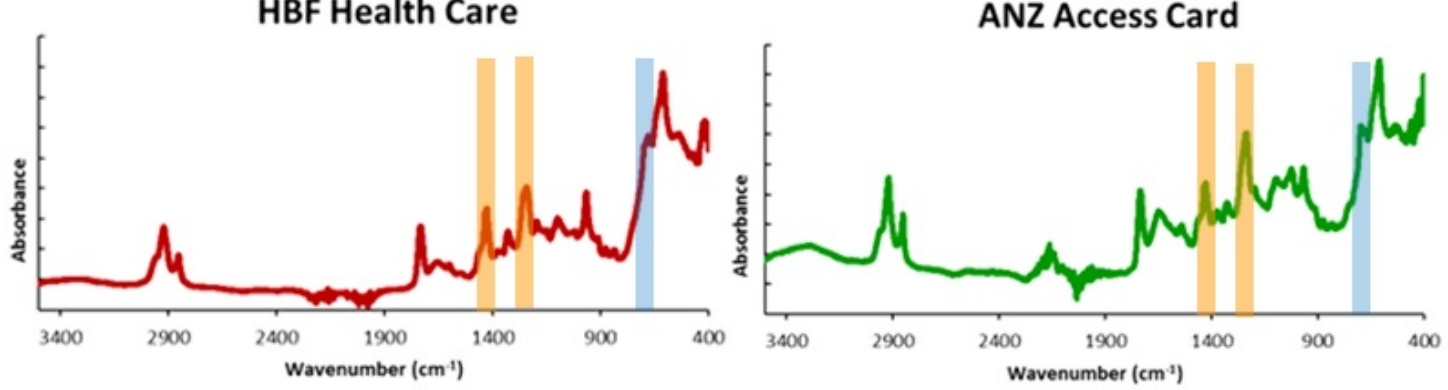

Saskatchewan Health Services
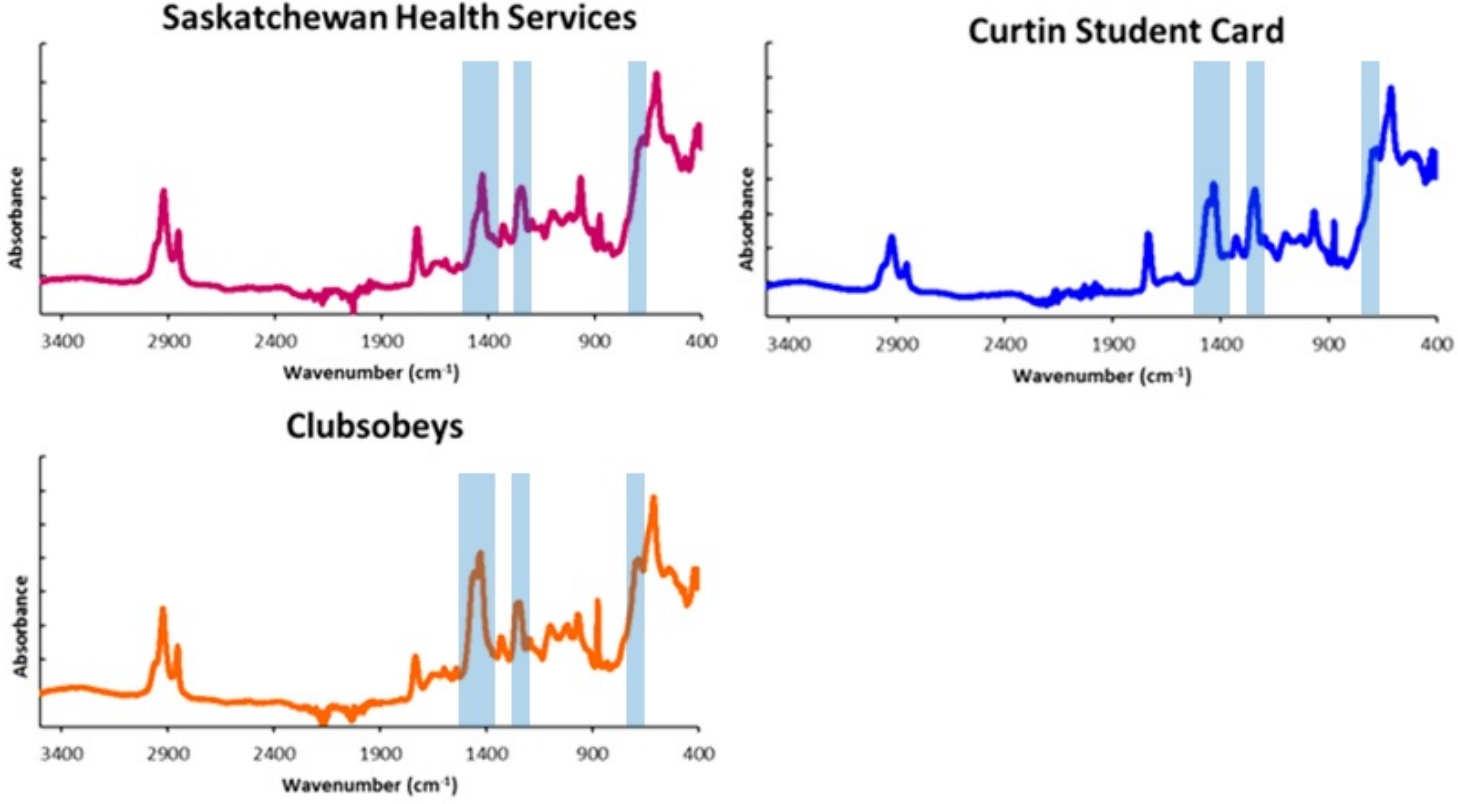

Figure 1: ATR-FTIR spectra of nine polymer card types. Blue highlighted regions are indicative of polyvinyl chloride and yellow regions are characteristic of polyethylene terephthalate. 
The observed spectral differences indicated the potential to separate these groups using chemometric methods. The spectra from each card were therefore subjected to principal component analysis (PCA) to reduce the data dimensionality. This is achieved by transforming the initial set of correlated variables (wavenumbers) into orthogonal variables known as principal components (PCs) [21, 22]. The samples can then be plotted using the first few PCs in order to visualise the sample distribution and identify latent groupings. The scores plot generated from this data is presented in Figure 2, which shows that separation of the groups occurs along PCs 1 and 2. Interestingly, the Western Australian driver's licence clustered with the ANZ, HBC and RAC cards along PC1, but with the remaining cards along PC2, reflecting compositional similarities to both groups. Although the driver's licence is thought to have a PVC core, it is possible that the laminate layers share a similar composition to the cards thought to consist of a PVC-PET blend.

The factor loadings for the first two PCs (Figure 3) were examined to identify spectral regions contributing to sample separation. Both components produced strong correlations against peaks in the alkyl C-H region. PC1 was positively correlated with $2930 \mathrm{~cm}^{-1}$ and $2900 \mathrm{~cm}^{-1}$ bands, while PC2 was negatively associated with $2920 \mathrm{~cm}^{-1}$ and $2850 \mathrm{~cm}^{-1}$ bands. This likely reflects a downshift in $\mathrm{C}-\mathrm{H}$ stretching frequencies in PET compared to PVC. As the lower stretching frequencies associated with PET are negatively correlated against PC2, it is expected that cards containing PET would obtain more negative scores against this component, which is reflected in the scores plot distribution. PC2 also has a strong negative correlation at ca. $1020 \mathrm{~cm}^{-1}$, reflecting possible variations in polymer chain length. The higher $\mathrm{C}-\mathrm{H}$ stretching frequencies likely associated with PVC are positively correlated with PC1, causing samples with a greater proportion of PVC to attain more positive scores along this component. This also aligns with a positive correlation between $\mathrm{PC} 1$ and the $1250 \mathrm{~cm}^{-1} \mathrm{C}-\mathrm{H}$ bend 
associated with PVC. However, it should be noted that as the specific composition of the cards is unknown, these assignments cannot be made with absolute certainty.
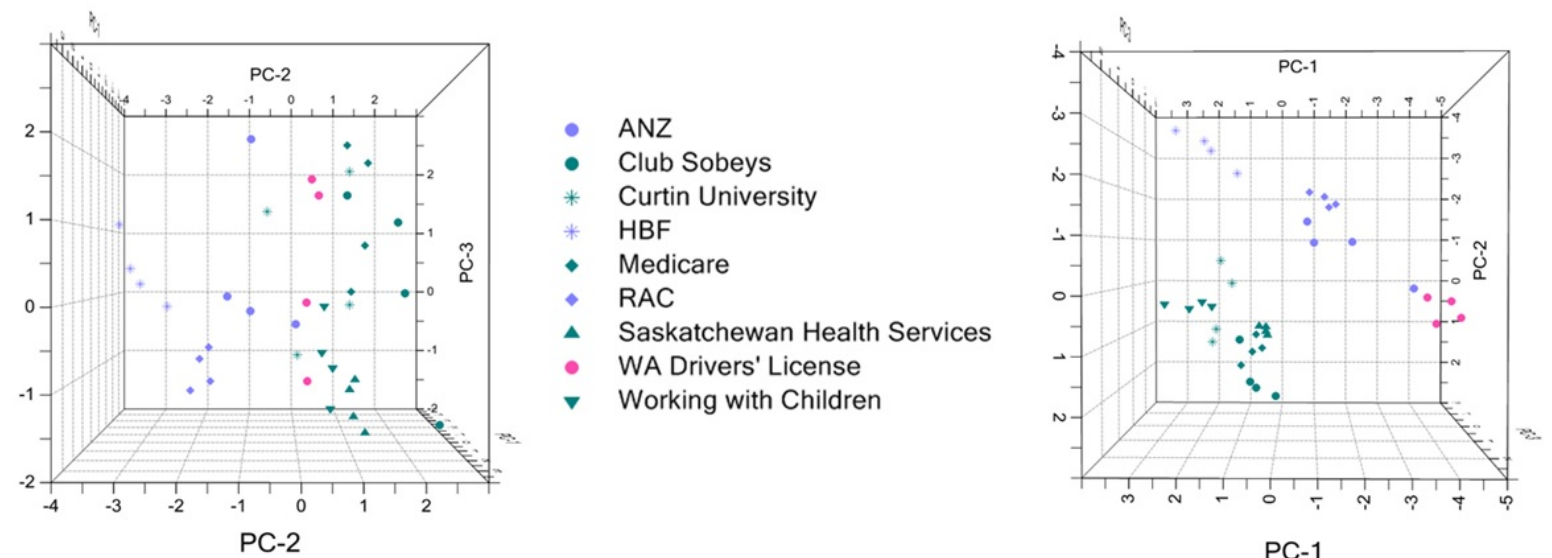

PC-1

Figure 2: Three-dimensional PCA scores plots of nine polymer card types based on their ATR-FTIR spectra. Left and right images show different perspectives of the same plot.

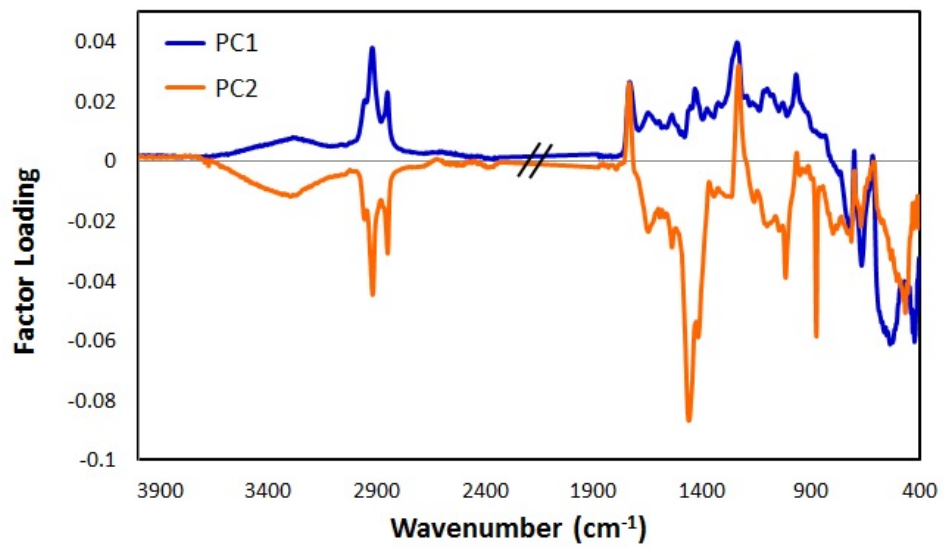

Figure 3: Factor loadings plot of PCs 1-2 for nine polymer card types based on their ATR-FTIR spectra.

\subsection{Discrimination and classification of Australian driver's licences}

Further work focussed on Western Australian driver's licence cards to determine whether separation between different versions of this card type could be achieved. Identity cards such as driver's licences are the most commonly detected form of fraudulent document; often used to facilitate organised crimes such as migrant smuggling or human trafficking $[1,2]$. Licence cards can also be used as "breeder" documents to obtain legitimate forms of identification, such as passports [6].

There are currently two series of driver's licence valid within Western Australia, which began distribution in 2011 and 2014. An expired series distributed from 2001-2011 was also investigated. PCA was carried out on fifteen licence cards, with $89.4 \%$ of total variance described by the first three PCs. The resulting scores plot (Figure 4a) showed a clear separation of the 2011 series along PC1, whilst the 2001 and 2014 series were overlapping. The 2014 series produced a high level of dispersion along PC3, suggesting that the composition of this series is potentially not as consistent 
between individual licence cards. Separate PCA of the 2001 and 2014 series cards resulted in some level of separation along PC1 and PC3 (Figure 4b). However, as the intra-group and inter-group variation were of similar magnitudes, these series were not considered to be reliably distinguished.

a)

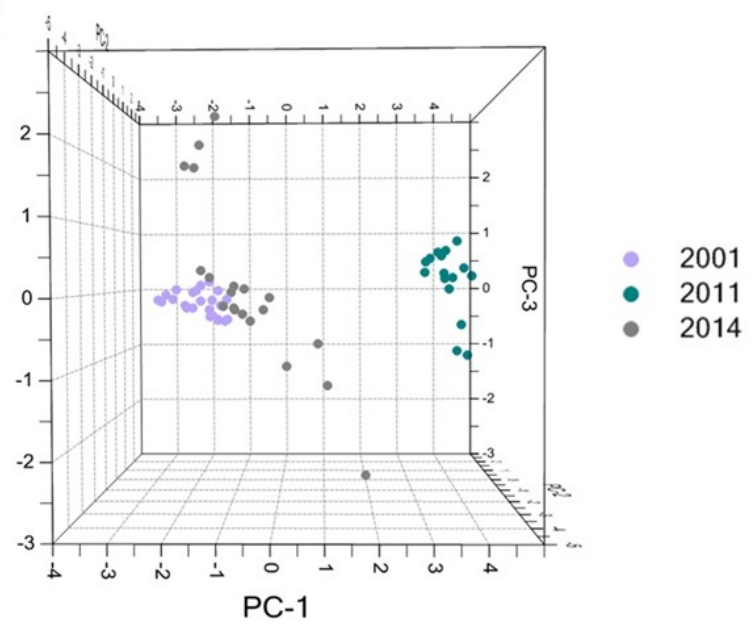

b)

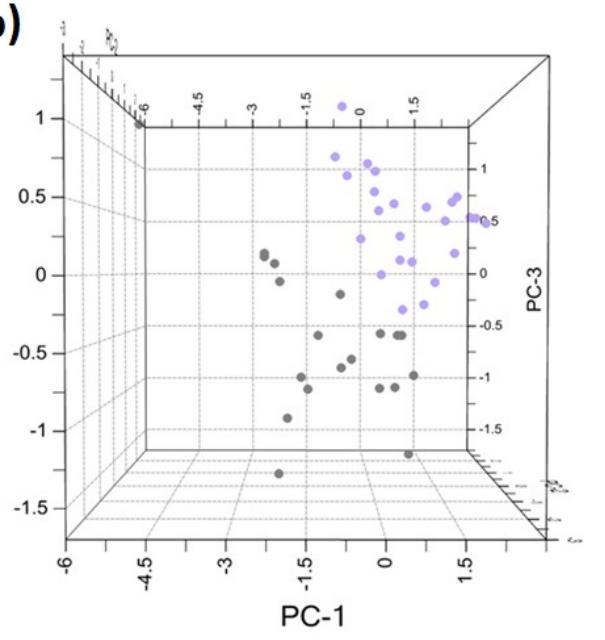

Figure 4: Three-dimensional PCA scores plots of (a) 2001, 2011 and 2014 series; and (b) 2001 and 2014 series Western Australian driver's licences based on their ATR-FTIR spectra.

Inspection of the PC1 factor loadings for analysis of all three series (Figure 5a) revealed strong positive correlations at ca. $2925 \mathrm{~cm}^{-1}$ and $1245 \mathrm{~cm}^{-1}$, along with negative correlations at $540 \mathrm{~cm}^{-1}$ and $425 \mathrm{~cm}^{-1}$. Comparing the normalised spectra for the centroid of each series (Figure 5b), the 2011 series has a greater relative absorbance at the $2925 \mathrm{~cm}^{-1}$ and $1245 \mathrm{~cm}^{-1}$ peaks, and much lower absorbance below $570 \mathrm{~cm}^{-1}$, resulting in more positive scores along PC1. This may reflect a greater relative proportion of PVC compared to other constituents in the non-core layers. PC3 was positively associated with the chain stretch region at $1020 \mathrm{~cm}^{-1}$, indicating that the dispersion amongst 2014 series cards along this component may be due to chain length variations. However, due to the layered nature and unspecified composition of these cards, it is again difficult to determine exact differences leading to separation. It should be emphasised that these spectral differences may not be readily identifiable to field personnel such as customs or licensing agents. The development of a chemometric model to flag suspect cards would thus be highly beneficial for frontline use. 
a)

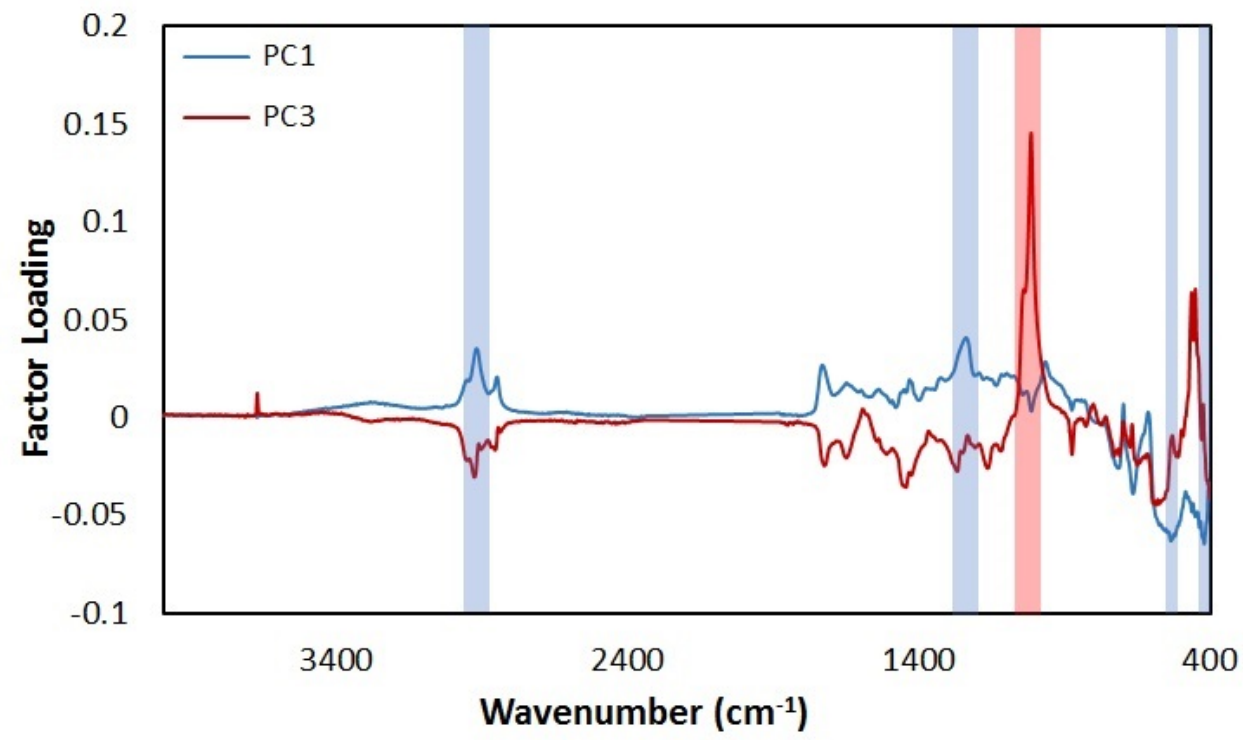

b)

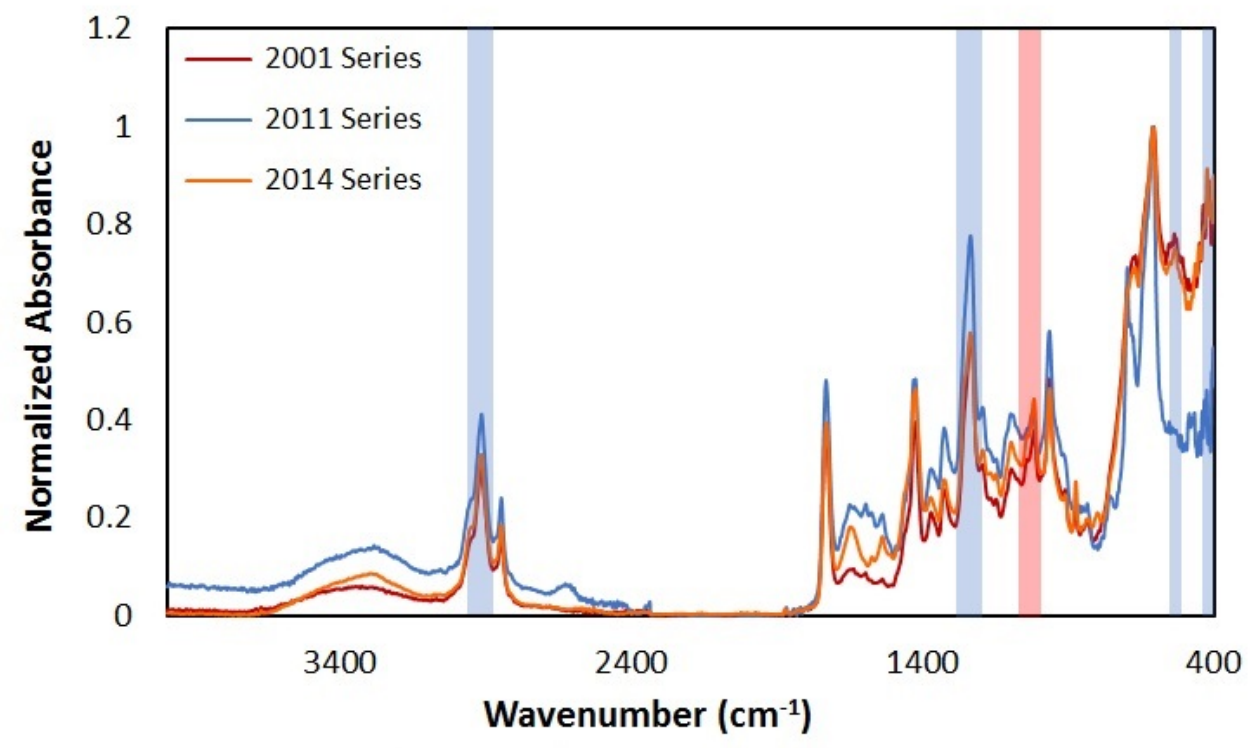

Figure 5: a) Factor loadings of PC1 and PC3 for three series of Western Australian driver's licences based on their ATR-FTIR spectra; and b) Normalized spectra from the centroid of each series.

A discriminant model was therefore constructed from the calibration set using the first four PCs, treating each series as a discrete class. The resulting model gave $98.3 \%$ correct classifications for the calibration set (Table 2). As expected, the only incorrect classification was between the 2001 and 2014 series. Development of a separate discriminant model incorporating only these two series did not alter this misclassification. Nonetheless, the incorrect classification was limited to a single replicate, and thus the overall assignment of this card remained correct.

Table 2: Classifications of samples in the calibration set using a four-PC LDA model. The overall classification accuracy was $98.3 \%$.

\begin{tabular}{lll}
\hline Series & Correct & Incorrect \\
\hline 2001 & 24 & 0 \\
2011 & 16 & 0 \\
2014 & 19 & $1(2001)$ \\
\hline
\end{tabular}


Using the same data to both construct and test the model can often result in an over-prediction of its efficacy. The model was hence further evaluated using a separate validation set of seven driver's licences not incorporated in the model. These included two 'atypical' cards that did not belong to any of the three series investigated; a pre-2001 series Western Australian licence and an interstate licence issued in New South Wales.

Of the five licences belonging to one of the series included in the model, four were correctly classified, with the single misclassification again occurring between the 2001 and 2014 series. The pre-2001 licence was predicted as belonging to the 2001 series, indicating that the composition did not significantly change when the 2001 series began distribution. The New South Wales licence was predicted as corresponding to a 2014 series Western Australian licence. As it is not known whether this licence in fact consists of the same polymer as the Western Australian 2014 series, it cannot be ascertained whether this is a 'correct' assignment.

Table 3: Classifications of samples in the validation set using a four-PC LDA model. Labels in brackets indicate assigned groups. N/A = Not Applicable.

\begin{tabular}{lll}
\hline Card Type & Actual Series & Predicted Series \\
\hline WA Driver's License (1999) & N/A & 2001 \\
WA Driver's License (2001) & 2001 & 2001 \\
WA Driver's License (2006) & 2001 & 2001 \\
WA Driver's License (2007) & 2001 & 2001 \\
WA Driver's License (2012) & 2011 & 2011 \\
WA Driver's License (2017) & 2014 & 2001 \\
NSW Driver's License & N/A & 2014 \\
\hline
\end{tabular}

It should be noted that when using LDA, all samples will be assigned to a pre-existing class. This assignment is made according to the sample's discriminant values (DVs), which act as distance measures between the sample and each known group. An unknown sample is classified as belonging to the group yielding the smallest magnitude discriminant value, indicative of the 'closest fit'. DVs for a correctly classified Western Australian driver's licence, incorrectly classified licence and the New South Wales licence are displayed in Table 4.

Table 4: Discriminant values for a correctly classified driver's licence, incorrectly classified driver's licence and an atypical licence using a four-PC LDA model. Values are averaged across four replicate spectra.

\begin{tabular}{llll}
\hline Licence & 2001 Series & 2011 Series & 2014 Series \\
\hline WA Driver's License (2017) & -2.1 & -66.3 & -8.1 \\
WA Driver's License (2012) & -83.4 & -3.0 & -63.9 \\
NSW Driver's License & -25.7 & -33.1 & -10.9 \\
\hline
\end{tabular}

The incorrectly classified Western Australian license obtained similar, low-magnitude values against both the 2001 series $(-2.1)$ and 2014 series (-8.1). The similar values indicate that the licence was actually placed in the region between the 2001 and 2014 groupings and is not well classified. The 
fact that both values are of low magnitude also indicates poor class separation, with a distance measure of only 10.2 between the centroids of each series. The New South Wales licence, although assigned to the 2014 series, similarly obtained similar magnitude values against all three series. Projection of these spectra onto the initial PCA model confirmed that this licence was placed between the three series and thus could not be confidently assigned (Figure S4).

Comparatively, the correctly classified licence obtained a small magnitude value against the correct 2011 series (-3.0) and much larger values against the remaining series, indicating that this licence is both well classified and distinctly separated from the remaining groups. In this way, atypical samples can be readily identified from those that are confidently assigned. These results highlight the importance of examining the discriminant values to evaluate the quality of predictions made using chemometric models.

\subsection{Synchrotron imaging}

To gain a better understanding of what might be causing the separation between licence series, an investigation into the structural differences was undertaken. This work was undertaken at the Infrared Microscopy (IRM) Beamline at the Australian Synchrotron, Melbourne. Thin cross-sections approximately $7 \mu \mathrm{m}$ thick were taken from a 2001 series Western Australian driver's licence for FTIR imaging (Figure S5). False-colour maps of the cross-sections were generated from selected integrated peaks, and then superimposed over an optical image of the sample to create a set of composite images (Figure 6).

Inspection of these images revealed a small feature in both cards which appears to be positioned between the primary layers. Spectra extracted from this feature exhibit peaks centred at ca. $2850 \mathrm{~cm}^{-1}, 1740 \mathrm{~cm}^{-1}$ and $1650 \mathrm{~cm}^{-1}$. These peaks were assigned as $\mathrm{C}-\mathrm{H}$ stretching, $\mathrm{C}=\mathrm{O}$ stretching and $\mathrm{N}-\mathrm{H}$ bending vibrations respectively. Based on the spectra, this feature is suspected to be a thin layer of adhesive such as polyester amide [23]. This indicates that discrimination between licences may be influenced by the use of a different adhesive between each licence series. 

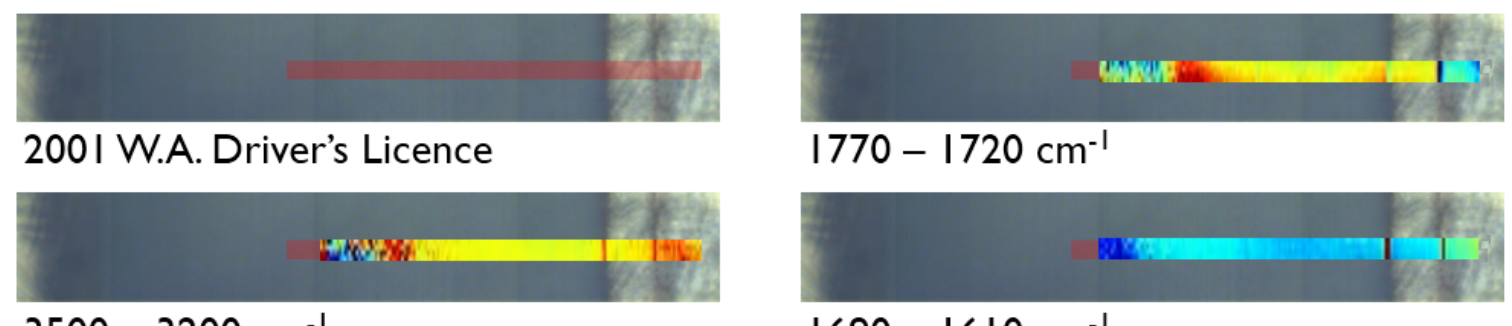

$$
1770-1720 \mathrm{~cm}^{-1}
$$
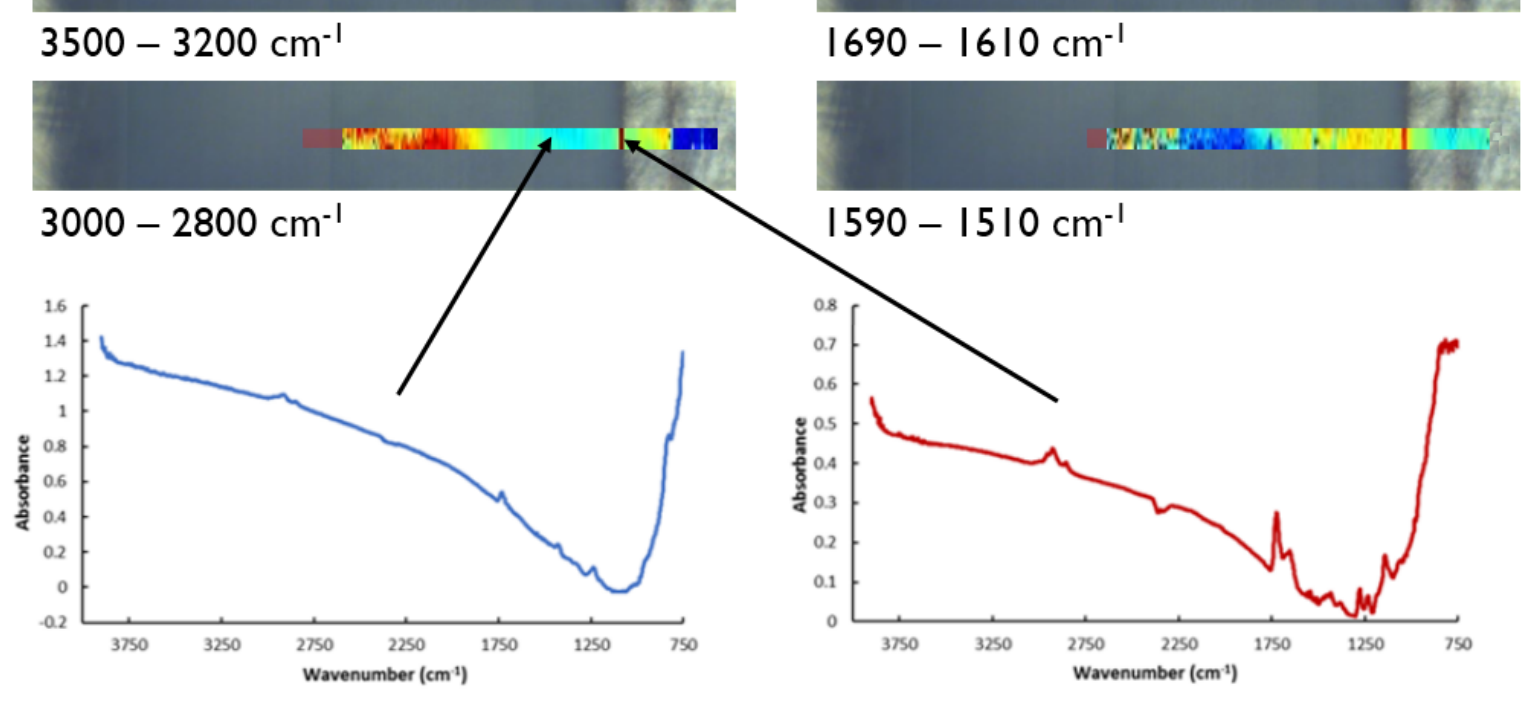

Figure 6: Composite FTIR false colour images from cross-sections of a 2001 series Western Australian driver's licence, based on integration of selected infrared peaks. Representative spectra are shown for the core polymer and suspected adhesive layer. Decreasing intensity from red to blue.

\section{Conclusions}

ATR-FTIR spectroscopy with chemometrics was successfully used to distinguish broad groupings of polymer cards based on their core composition. Similarly, a focussed investigation of Western Australian driver's licence cards resulted in clear discrimination between the two currently valid series (2011 and 2014). A subsequent predictive model was able to correctly classify four of five test licences, and atypical or poorly classified licences were readily identifiable from their discriminant values. The developed model may be used as a simple and high-throughput screening method for 'frontline' personnel to identify cards that have a composition or structure that is inconsistent with their purported type and origin. This would enable more rapid and accurate referral of potential counterfeits to a document examiner for further examination. This approach could also provide document examiners with a statistically validated basis on which to support their conclusions from visual examinations.

Synchrotron FTIR imaging identified a potential adhesive layer, suspected to be a polyester amide, which could potentially be contributing to separation between different cards. However, this requires confirmation through analysis of additional samples. It would also be of interest to conduct Raman imaging as a complementary technique to FTIR, allowing a more complete chemical characterisation to be obtained.

Transitioning this approach to operational use will first require further validation using a wider range of cards, including known counterfeits. Development of an appropriate sample mount for the collection of edge spectra would also improve the reproducibility of spectral measurements, 
potentially allowing for more reliable separation between groups. Future work may also examine the effect of card storage conditions on their characterisation using spectroscopic techniques.

\section{Funding}

We acknowledge travel funding provided by the Synchrotron Access Program, managed by the Australian Synchrotron, part of ANSTO, and funded by the Australian Government.

\section{Acknowledgements}

Portions of this research were undertaken on the Infrared Microspectroscopy Beamline at the Australian Synchrotron, Victoria, Australia; part of Australia's Nuclear Science and Technology Organisation (ANSTO). The authors would like to especially acknowledge the technical support provided by Dr Jitraporn Vongsvivut, Dr Mark Tobin, Dr Keith Bambery and Dr Donna Menzies (Australian Synchrotron) as well as Peter Chapman (Curtin University). 


\section{References}

1. Europol, European Union Serious and Organised Crime Threat Assessment: Crime in the Age of Technology. 2017, Europol: The Hague, Netherlands.

2. UNODC, Handbook on Identity-related Crime. 2011, United Nations: Vienna, Austria.

3. Yesildag, C. and G. Tziovaras. Polycarbonate Films - Material For Intelligent, High-Quality Cards. 08-09-16]; Available from:

http://www.icma.com/ArticleArchives/Polycarbonate5605.htm.

4. Rockwell, L.R., Payment Cards, in Encyclopedia of Forensic Sciences (Second Edition), J.A. Siegel, P.J. Saukko, and M.M. Houck, Editors. 2013, Academic Press: Waltham. p. 432-438.

5. ABNote. Identity card solutions. 2014; Available from: http://www.abnote.com.au/sites/default/files/Identity Card Solutions.pdf.

6. Trubshoe, T. and J. McGinn, Forgery/Counterfeits, in Encyclopedia of Forensic Sciences, M.M. Houck, J.A. Siegel, and P.J. Saukko, Editors. 2013, Academic Press: Waltham. p. 360-366.

7. Jones, R.L., et al., Contact smart cards having a document core, contactless smart cards including multi-layered structure, PET-based identification document, and methods of making same, C.I.P. Office, Editor. 2016: Canada.

8. Morrill Webb, L.A. and W.J. Faenza, Transaction card, U.S. Patent, Editor. 2012, III Holdings 1., LLC: United States.

9. Tiguy, A.S.R., D. Bi, and R. Jones, Identification document with tactile feature, U.S. Patent, Editor. 2018, MorphoTrust USA, LLC United States.

10. Gariup, M. and J. Piskorski, The challenge of detecting false documents at the border: Exploring the performance of humans, machines and their interaction. International Journal of Critical Infrastructure Protection, 2018.

11. Maric, M., et al., Rapid characterisation and classification of automotive clear coats by attenuated total reflectance infrared spectroscopy. Analytical Methods, 2012. 4(9): p. 26872693.

12. Sauzier, G., et al., Preliminary studies into the effect of environmental degradation on the characterisation of automotive clear coats by attenuated total reflectance infrared spectroscopy. Analytical Methods, 2013. 5(19): p. 4984-4990.

13. Holman, S.W., T.F. Emmett, and M.D. Cole, A quantitative assessment of the chemical variation in food grade polyethylene cling film, a common wrapping material for illicit drugs, using attenuated total reflection-Fourier transform infrared spectroscopy. Analytical Methods, 2012. 4(6): p. 1667-1673.

14. Telford, C.J., et al., Classification of polyethylene cling films by attenuated total reflectanceFourier transform infrared spectroscopy and chemometrics. Analytical Methods, 2017. 9(2): p. 192-197.

15. Larsen, R.A., et al., FT-IR microscopic analysis of polymer laminate samples including transmission and ATR spectroscopy. Spectroscopy, 2016. 31(8): p. 16-23.

16. Široká, B., J. Široký, and T. Bechtold, Application of ATR-FT-IR single-fiber analysis for the identification of a foreign polymer in textile matrix. International Journal of Polymer Analysis and Characterization, 2011. 16(4): p. 259-268.

17. Pumure, I., et al., Analysis of ATR-FTIR absorption-reflection data from 13 polymeric fabric materials using chemometrics. American Journal of Analytical Chemistry, 2015. 6(4): p. 305312.

18. Liang, C.Y. and S. Krimm, Infrared spectra of high polymers: Part IX. Polyethylene terephthalate. Journal of Molecular Spectroscopy, 1959. 3(1): p. 554-574.

19. Stormberg, R.R., S. Straus, and B.G. Achhammer, Infrared spectra of thermally degraded poly(vinyl chloride $\}^{*}$. Journal of Research of the National Bureau of Standards, 1958. 60(2): p. 147-152. 
20. Pavia, D.L., et al., Introduction to Spectroscopy. 5th ed. ed. 2015, Stanford, U.S.: Cengage Learning.

21. Brereton, R.G., Pattern recognition in chemometrics. Chemometrics and Intelligent Laboratory Systems, 2015. 149B: p. 90-96.

22. Abdi, H. and L.J. Williams, Principal component analysis. Wiley Interdisciplinary Reviews: Computational Statistics, 2010. 2(4): p. 433-459.

23. Kadam, P.G., P. Vaidya, and S.T. Mhaske, Synthesis and characterization of polyesteramide hot melt adhesive from low purity dimer acid, ethylenediamine, and ethanolamine. Journal of Polymers, 2014: p. 1-8. 


\section{ATR-FTIR spectroscopic studies of polymer-based identification cards}

Jasmine McGann ${ }^{a, b \neq}$, Georgina Sauzier ${ }^{\mathrm{a}, \mathrm{b}}$, Mark J. Hackett ${ }^{\mathrm{a}, \mathrm{b}}$, Wilhelm van Bronswijk ${ }^{\mathrm{a}}$, Tonya Trubshoe ${ }^{c}$, John McGinnc and Simon W. Lewis ${ }^{a, b^{*}}$

a School of Molecular and Life Sciences, Curtin University, GPO Box U1987, Perth, Western Australia, 6845, Australia.

${ }^{b}$ Curtin Institute of Functional Molecules and Interfaces, Curtin University, GPO Box U1987, Perth, Western Australia, 6845, Australia.

c Document Examination Solutions, PO Box 7317, Karawara, Western Australia, 6152, Australia.

¥ Present address: ChemCentre, PO Box 1250, Bentley, Western Australia, 6983, Australia.

\section{Supplementary Information}

All data associated with this work is available open-access via Research Data Australia;

DOI: $10.25917 / 5$ bee6a54e0ead

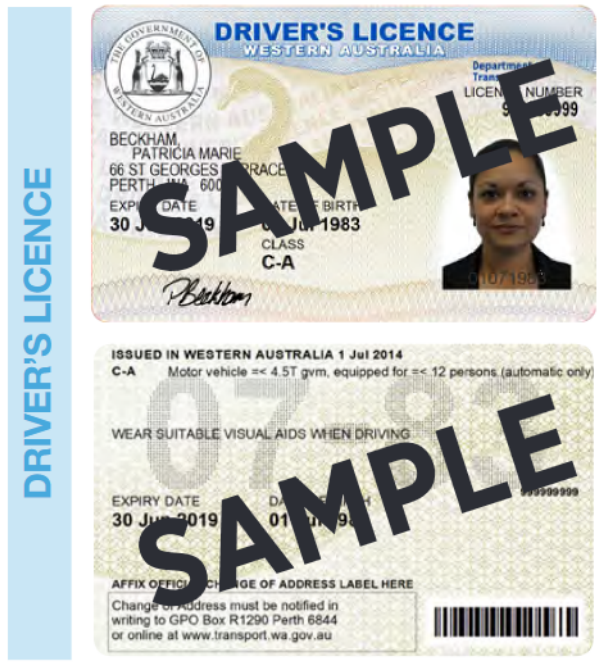

2001 Series
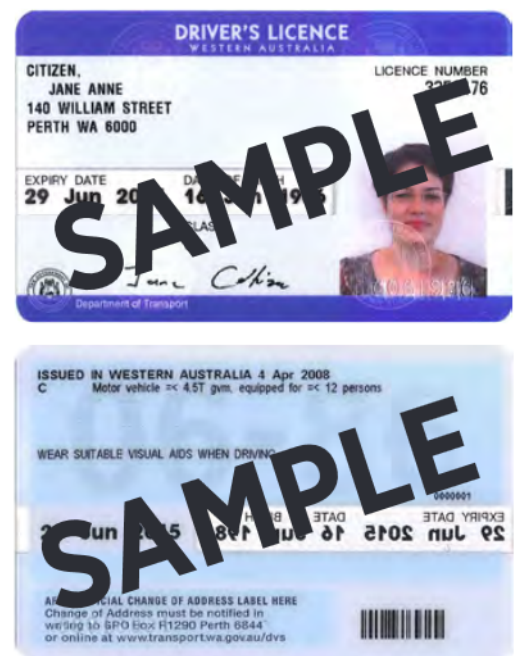

2011 Series
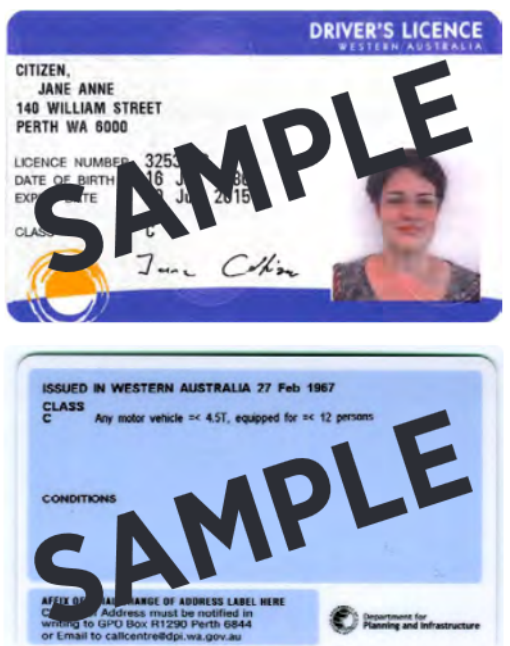

2014 Series

Figure S1: Sample images of Western Australia driver's licence series used in this study. Image adapted from Western Australia Department of Transport [1]. 


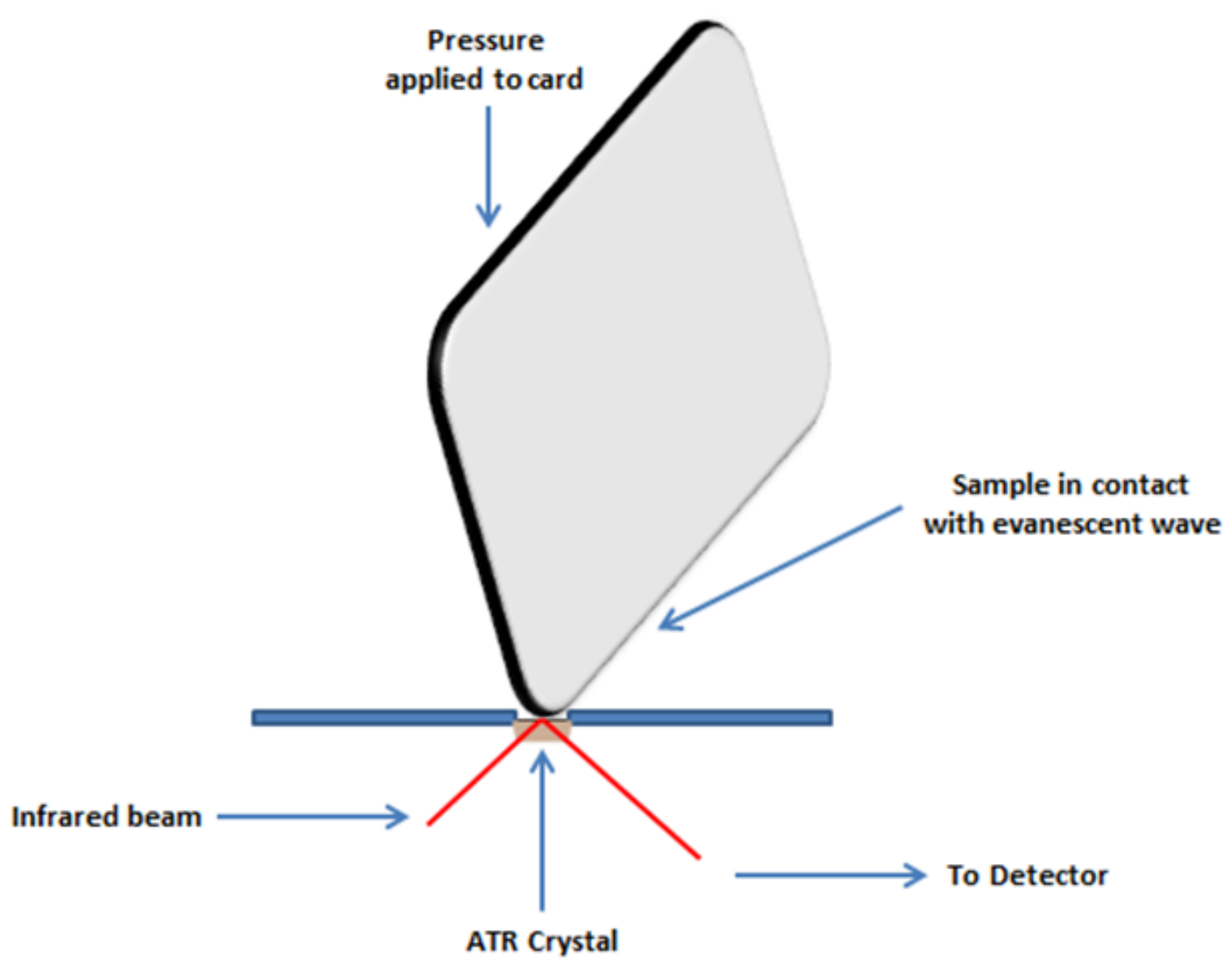

Figure S2: Measurement set-up for cross-sectional ATR-FTIR analysis of polymer cards.

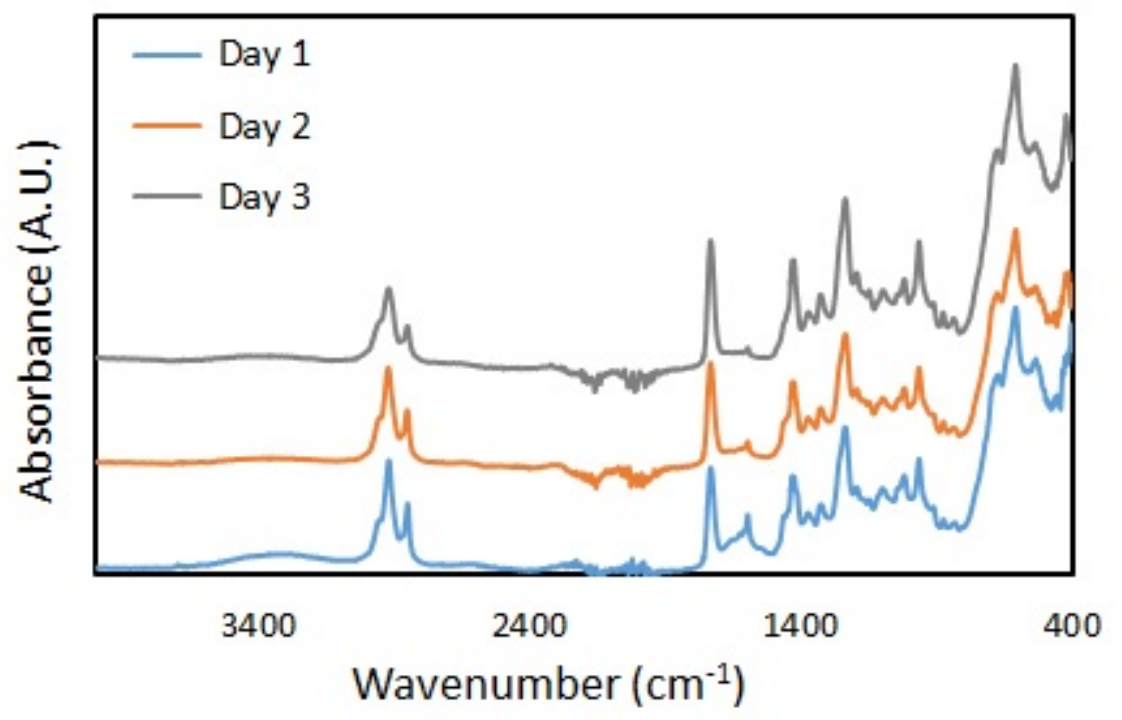

Figure S3: Replicate ATR-FTIR measurements acquired from a 2001 series Western Australian driver's licence across three different days. Spectra have been offset for clarity. 


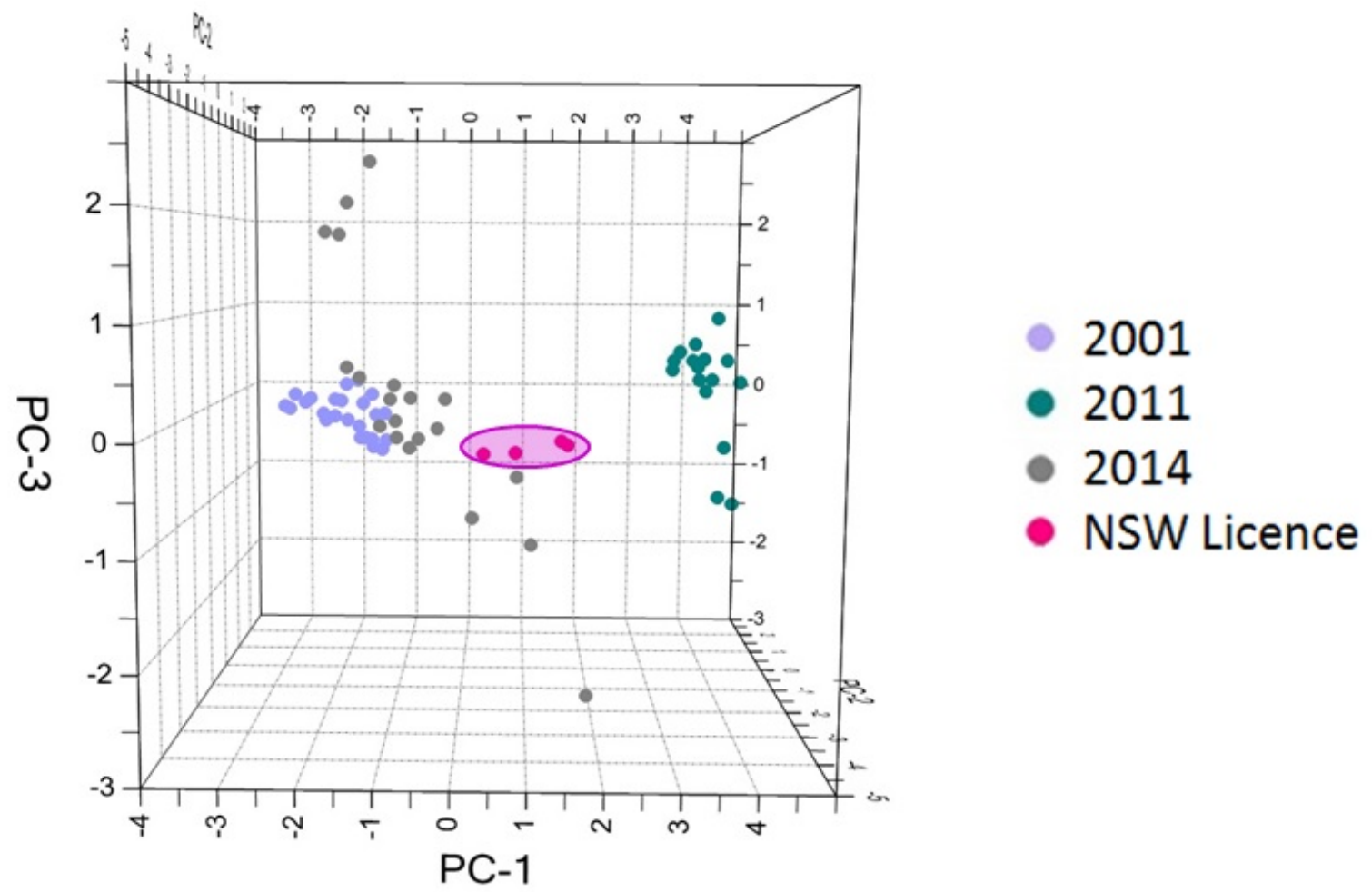

Figure S4: Three-dimensional PCA scores plot showing projection of spectra collected from a New South Wales driver's licence (circled) onto the PCA model constructed using Western Australian driver's licences.

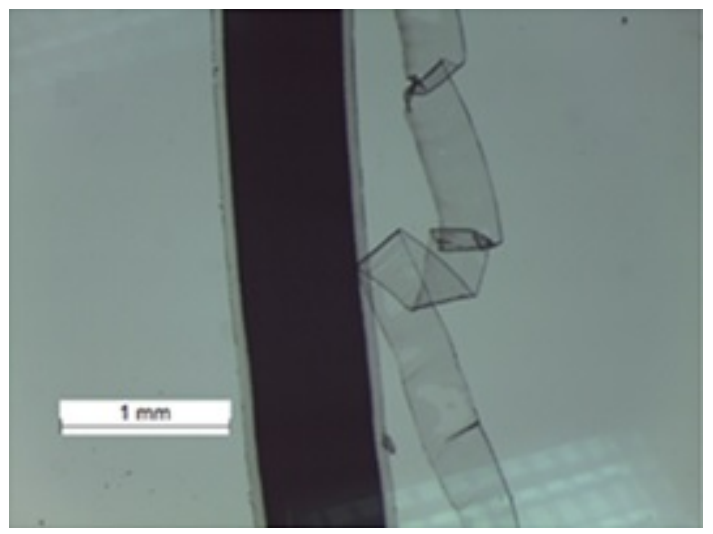

Figure S5: Microtomed cross-section of a 2001 series Western Australian driver's licence.

\section{References}

1. Department of Transport. Increased security of licence cards. 2017; Available from: https://www.transport.wa.gov.au/licensing/drivers-licence-cards.asp. 

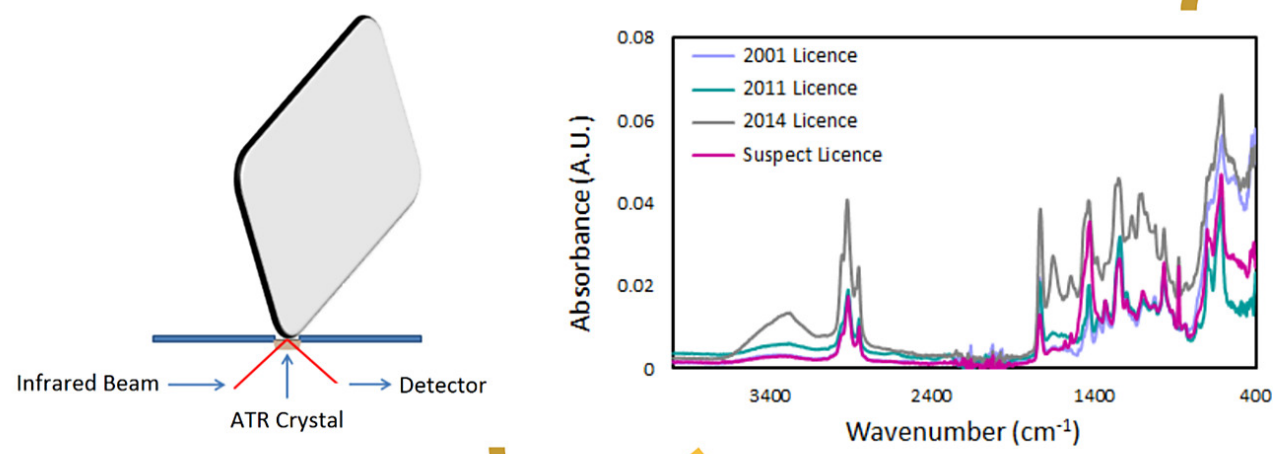

COUNTERFET

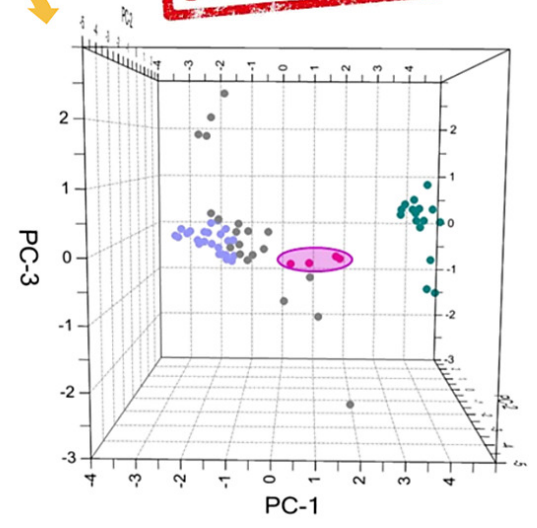

